\title{
Rule Discovery in Large Time-Series Medical Databases
}

\author{
Shusaku Tsumoto \\ Department of Medicine Informatics, Shimane Medical University, School of Medicine, \\ 89-1 Enya-cho Izumo City, Shimane 693-8501 Japan \\ E-mail: tsumoto@computer.org
}

\begin{abstract}
Since hospital information systems have been introduced in large hospitals, a large amount of data, including laboratory examinations, have been stored as temporal databases. The characteristics of these temporal databases are: (1) Each record are inhomogeneous with respect to time-series, including short-term effects and long-term effects. (2) Each record has more than 1000 attributes when a patient is followed for more than one year. (3) When a patient is admitted for a long time, a large amount of data is stored in a very short term. Even medical experts cannot deal with these large databases, the interest in mining some useful information from the data are growing. In this paper, we introduce a combination of extended moving average method and rule induction method, called CEARI to discover new knowledge in temporal databases. This CEARI was applied to a medical dataset on Motor Neuron Diseases, the results of which show that interesting knowledge is discovered from each database.
\end{abstract}

\section{Introduction}

Since hospital information systems have been introduced in large hospitals, a large amount of data, including laboratory examinations, have been stored as temporal databases 11. For example, in a university hospital, where more than 1000 patients visit from Monday to Friday, a database system stores more than 1 GB numerical data of laboratory examinations. Thus, it is highly expected that data mining methods will find interesting patterns from databases because medical experts cannot deal with those large amount of data. The characteristics of these temporal databases are: (1) Each record are inhomogeneous with respect to time-series, including short-term effects and long-term effects. (2) Each record has more than 1000 attributes when a patient is followed for more than one year. (3) When a patient is admitted for a long time, a large amount of data is stored in a very short term. Even medical experts cannot deal with these large temporal databases, the interest in mining some useful information from the data are growing.

In this paper, we introduce a combination of extended moving average method and rule induction method, called CEARI to discover new knowledge in temporal databases. In the system, extended moving average method are used 
for preprocessing, to deal with irregularity of each temporal data. Using several parameters for time-scaling, given by users, this moving average method generates a new database for each time scale with summarized attributes. Then, rule induction method is applied to each new database with summarized attributes. This CEARI was applied to two medical datasets, the results of which show that interesting knowledge is discovered from each database.

\section{Temporal Databases in Hospital Information Systems}

Since incorporating temporal aspects into databases is still an ongoing research issue in database area [1], temporal data are stored as a table in hospital information systems(H.I.S.). Table 1 shows a typical example of medical data, which is retrieved from H.I.S. The first column denotes the ID number of each patient, and the second one denotes the date when the datasets in this row is examined. Each row with the same ID number describes the results of laboratory examinations, which were taken on the date in the second column. For example, the second row shows the data of the patient ID 1 on $04 / 19 / 1986$. This simple database show the following characteristics of medical temporal database: (1)The Number of Attributes are too many. Even though the dataset of a patient focuses on the transition of each examination (attribute), it would be difficult to see its trend when the patient is followed for a long time. If one wants to see the long-term interaction between attributes, it would be almost impossible. In order to solve this problems, most of H.I.S. systems provide several graphical interfaces to capture temporal trends 11. However, the interactions among more than three attributes are difficult to be studied even if visualization interfaces are used. (2)Irregularity of Temporal Intervals. Temporal intervals are irregular. Although most of the patients will come to the hospital every two weeks or one month, physicians may not make laboratory tests at each time. When a patient has a acute fit or suffers from acute diseases, such as pneumonia, laboratory examinations will be made every one to three days. On the other hand, when his/her status is stable, these test may not be made for a long time. Patient ID 1 is a typical example. Between 04/30 and 05/08/1986, he suffered from a pneumonia and was admitted to a hospital. Then, during the therapeutic procedure, laboratory tests were made every a few days. On the other hand, when he was stable, such tests were ordered every one or two year. (3)Missing Values. In addition to irregularity of temporal intervals, datasets have many missing values. Even though medical experts will make laboratory examinations, they may not take the same tests in each instant. Patient ID 1 in Table 1 is a typical example. On 05/06/1986, medical physician selected a specific test to confirm his diagnosis. So, he will not choose other tests. On 01/09/1989, he focused only on GOT, not other tests. In this way, missing values will be observed very often in clinical situations.

These characteristics have already been discussed in KDD area [5]. However, in real-world domains, especially domains in which follow-up studies are crucial, such as medical domains, these ill-posed situations will be distinguished. If one 
Table 1. An Example of Temporal Database

\begin{tabular}{lccccccccc}
\multicolumn{1}{l}{ ID } & Date & GOT GPT LDH & $\gamma$-GTP TP edema & $\cdots$ \\
\hline 1 & 19860419 & 24 & 12 & 152 & 63 & 7.5 & - & $\cdots$ \\
1 & 19860430 & 25 & 12 & 162 & 76 & 7.9 & + & $\cdots$ \\
1 & 19860502 & 22 & 8 & 144 & 68 & 7.0 & + & $\cdots$ \\
1 & 19860506 & & & & & & & $\cdots$ \\
1 & 19860508 & 22 & 13 & 156 & 66 & 7.6 & - & $\cdots$ \\
1 & 19880826 & 23 & 17 & 142 & 89 & 7.7 & - & $\cdots$ \\
1 & 19890109 & 32 & & & & & - & $\cdots$ \\
1 & 19910304 & 20 & 15 & 369 & 139 & 6.9 & + & $\cdots$ \\
\hline 2 & 19810511 & 20 & 15 & 369 & 139 & 6.9 & - & $\cdots$ \\
2 & 19810713 & 22 & 14 & 177 & 49 & 7.9 & - & $\cdots$ \\
2 & 19880826 & 23 & 17 & 142 & 89 & 7.7 & - & $\cdots$ \\
2 & 19890109 & 32 & & & & & - & $\cdots$ \\
\hline
\end{tabular}

wants to describe each patient (record) as one row, then each row have too many attributes, which depends on how many times laboratory examinations are made for each patient. It is notable that although the above discussions are made according to the medical situations, similar situations may occur in other domains with long-term follow-up studies.

\section{$3 \quad$ Extended Moving Average Methods}

\subsection{Moving Average Methods}

Averaging mean methods have been introduced in statistical analysis [6]. Temporal data often suffers from noise, which will be observed as a spike or sharp wave during a very short period, typically at one instant. Averaging mean methods remove such an incidental effect and make temporal sequences smoother.

With one parameter $w$, called window, moving average $\hat{y}_{w}$ is defined as follows:

$$
\hat{y}_{w}=\sum_{j=1}^{w} y_{j} .
$$

For example, in the case of GOT of patient ID 1, $y_{5}$ is calculated as: $\hat{y}_{5}=$ $(24+25+22+22+22) / 5=23.0$. It is easy to see that $\hat{y}_{w}$ will remove the noise effect which continue less than $w$ points.

The advantage of moving average method is that it enables to remove the noise effect when inputs are given periodically [6]. For example, when some tests are measured every several days 1 , the moving average method is useful to remove the noise and to extract periodical domains. However, in real-world domains,

\footnotetext{
1 This condition guarantees that measurement is approximately continuous
} 
inputs are not always periodical, as shown in Table 1. Thus, when applied timeseries are irregular or discrete, ordinary moving average methods are powerless. Another disadvantage of this method is that it cannot be applicable to categorical attributes. In the case of numerical attributes, average can be used as a summarized statistic. On the other hand, such average cannot be defined for categorical attributes.

Thus, we introduce the extended averaging method to solve these two problems in the subsequent subsections.

\subsection{Extended Moving Average for Continuous Attributes}

In this extension, we first focus on how moving average methods remove noise. The key idea is that a window parameter $w$ is closely related with periodicity. If $w$ is larger, then the periodical behavior whose time-constant is lower than $w$ will be removed. Usually, a spike by noise is observed as a single event and this effect will be removed when $w$ is taken as a large value. Thus, the choice of $w$ separates different kinds of time-constant behavior in each attribute and in the extreme case when $w$ is equal to total number of temporal events, all the temporal behavior will be removed. We refer to this extreme case as $w=\infty$.

The extended moving average method is executed as follows: first calculates $y_{\infty}$ for an attribute $y$. Second, the method outputs its maximum and minimum values. Then, according to the selected values for $w$, a set of sequence $\left\{y_{w}(i)\right\}$ for each record is calculated. For example, if $\{w\}$ is equal to $\{10$ years, 5 years, 1 year, 3 months, 2 weeks $\}$, then for each element in $\{w\}$, the method uses the time-stamp attribute for calculation of each $\left\{y_{w}(i)\right\}$ in order to deal with irregularities. In the case of Table 1 , when $w$ is taken as 1 year, all the rows are aggregated into several components as shown in Table 2. From this aggregation, a sequence $y_{w}$ for each attribute is calculated as in Table 3 .

Table 2. Aggregation for $w=1$ (year)

\begin{tabular}{cccccccccc}
\multicolumn{1}{l}{ ID } & Date & \multicolumn{1}{c}{ GOT GPT LDH } & $\gamma$-GTP TP edema & $\cdots$ \\
\hline 1 & 19860419 & 24 & 12 & 152 & 63 & 7.5 & - & $\cdots$ \\
1 & 19860430 & 25 & 12 & 162 & 76 & 7.9 & + & $\cdots$ \\
1 & 19860502 & 22 & 8 & 144 & 68 & 7.0 & + & $\cdots$ \\
1 & 19860506 & & & & & & & $\cdots$ \\
1 & 19860508 & 22 & 13 & 156 & 66 & 7.6 & - & $\cdots$ \\
\hline 1 & 19880826 & 23 & 17 & 142 & 89 & 7.7 & - & $\cdots$ \\
\hline 1 & 19890109 & 32 & & & & & - & $\cdots$ \\
\hline \hline 1 & 19910304 & 20 & 15 & 369 & 139 & 6.9 & + & $\cdots$ \\
\hline
\end{tabular}


Table 3. Moving Average for $w=1$ (year)

\begin{tabular}{ccccccccc}
\multicolumn{1}{c}{ ID Period GOT GPT LDH } & $\gamma$-GTP & TP & edema & $\cdots$ \\
\hline 1 & 1 & 23.25 & 11.25 & 153.5 & 68.25 & 7.5 & $?$ & $\cdots$ \\
1 & 2 & 23 & 17 & 142 & 89 & 7.7 & $?$ & $\cdots$ \\
1 & 3 & 32 & & & & & $?$ & $\cdots$ \\
1 & 4 & & & & & & $?$ & $\cdots$ \\
1 & 5 & 20 & 15 & 369 & 139 & 6.9 & $?$ & $\cdots$ \\
\hline 1 & $\infty$ & 24 & 12.83 & 187.5 & 83.5 & 7.43 & $?$ & $\cdots$ \\
\hline & $\ldots$ & & & & & & &
\end{tabular}

\subsection{Categorical Attributes}

One of the disadvantages of moving average method is that it cannot deal with categorical attributes. To solve this problem, we will classify categorical attributes into three types, whose information should be given by users. The first type is constant, which will not change during the follow-up period. The second type is ranking, which is used to rank the status of a patient. The third type is variable, which will change temporally, but ranking is not useful. For the first type, extended moving average method will not be applied. For the second one, integer will be assigned to each rank and extended moving average method for continuous attributes is applied. On the other hand, for the third one, the temporal behavior of attributes is transformed into statistics as follows.

First, the occurence of each category (value) is counted for each window. For example, in Table 2, edema is a binary attribute and variable. In the first window, an attribute edema takes $\{-,+,+,-\} 2$ So, the occurence of - and + are 2 and 2 , respectively. Then, each conditional probability will be calculated. In the above example, probabilities are equal to $p\left(-\mid w_{1}\right)=2 / 4$ and $p\left(+\mid w_{1}\right)=2 / 3$. Finally, for each probability, a new attribute is appended to the table (Table 4 ).

Table 4. Final Table with Moving Average for $w=1$ (year)

\begin{tabular}{cccccccccc}
\multicolumn{1}{l}{ ID Period GOT } & GPT LDH & $\gamma$-GTP & TP & edema $(+)$ & edema $(-)$ & $\cdots$ \\
\hline 1 & 1 & 23.25 & 11.25 & 153.5 & 68.25 & 7.5 & 0.5 & 0.5 & $\cdots$ \\
1 & 2 & 23 & 17 & 142 & 89 & 7.7 & 0.0 & 1.0 & $\cdots$ \\
1 & 3 & 32 & & & & & 0.0 & 1.0 & $\cdots$ \\
1 & 4 & & & & & & 0.0 & 1.0 & $\cdots$ \\
1 & 5 & 20 & 15 & 369 & 139 & 6.9 & 1.0 & 0.0 & $\cdots$ \\
\hline 1 & $\infty$ & 24 & 12.83 & 187.5 & 83.5 & 7.43 & 0.43 & 0.57 & $\cdots$ \\
\hline
\end{tabular}

\footnotetext{
${ }^{2}$ Missing values are ignored for counting.
} 
Summary of Extended Moving Average. All the process of extended moving average is used to construct a new table for each window parameter as the first preprocessing. Then, second preprocessing method will be applied to newly generated tables. The first preprocessing method is summarized as follows.

1. Repeat for each $w$ in List $L_{w}$,

a) Select an attribute in a List $L_{a}$;

i. If an attribute is numerical, then calculate moving average for $w$;

ii. If an attribute is constant, then break;

iii. If an attribute is rank, then assign integer to each ranking; calculate moving average for $w$;

iv. If an attribute is variable, calculate frequency of each category;

b) If $L_{a}$ is not empty, goto (a).

c) Construct a new table with each moving average.

2. Construct a table for $w=\infty$.

\section{Second Preprocessing and Rule Discovery}

\subsection{Summarizing Temporal Sequences}

From the data table after processing extended moving average methods, several preprocessing methods may be applied in order for users to detect the temporal trends in each attribute. One way is discretization of time-series by clustering introduced by Das 4]. This method transforms time-series into symbols representing qualitative trends by using a similarity measure. Then, time-series data is represented as a symbolic sequence. After this preprocessing, rule discovery method is applied to this sequential data. Another way is to find auto-regression equations from the sequence of averaging means. Then, these quantitative equations can be directly used to extract knowledge or their qualitative interpretation may be used and rule discovery [3], other machine learning methods [7, or rough set method 9 can be applied to extract qualitative knowledge.

In this research, we adopt two modes and transforms databases into two forms: one mode is applying temporal abstraction method [8] as second preprocessing and transforms all continuous attributes into temporal sequences. The other mode is applying rule discovery to the data after the first preprocessing without second one. The reason why we adopted these two mode is that we focus not only on temporal behavior of each attribute, but also on association among several attributes. Although Miksch's method[8] and Das's approach[4] are very efficient to extract knowledge about transition, they cannot focus on association between attributes in an efficient way. For the latter purpose, much simpler rule discovery algorithm are preferred.

\subsection{Continuous Attributes and Qualitative Trend}

To characterize the deviation and temporal change of continuous attributes, we introduce standardization of continuous attributes. For this, we only needs the 
total average $\hat{y}_{\infty}$ and its standardization $\sigma_{\infty}$. With these parameters, standardized value is obtained as:

$$
z_{w}=\frac{y_{w}-\hat{y}_{\infty}}{\sigma_{\infty}}
$$

The reason why standardization is introduced is that it makes comparison between continuous attributes much easier and clearer, especially, statistic theory guarantees that the coefficients of a auto-regression equation can be compared with those of another equation [6].

After the standardization, an extraction algorithm for qualitative trends is applied 8]. This method is processed as follows: First, this method uses data smoothing with window parameters. Secondly, smoothed values for each attributes are classified into seven categories given as domain knowledge about laboratory test values: extremely low, substantially low, slightly low, normal range, slightly high, substantially high, and extremely high. With these categories, qualitative trends are calculated and classified into the following ten categories by using guideline rules: decrease too fast(A1), normal decrease(A2), decrease too slow (A3), zero change(ZA), dangerous increase(C), increase too fast(B1), normal increase(B2), increase too slow(B3), dangerous decrease(D). For example, if the value of some laboratory tests change from substantially high to normal range within a very short time, the qualitative trend will be classified into A1(decrease too fast). For further information, please refer to [8].

\subsection{Rule Discovery Algorithm}

For rule discovery, a simple rule induction algorithm discussed in [10] is applied, where continuous attributes are transformed into categorical attributes with a cut-off point. As discussed in Section 3, moving average method will remove the temporal effect shorter than a window parameter. Thus, $w=\infty$ will remove all the temporal effect, so this moving average can be viewed as data without any temporal characteristics. If rule discovery is applied to this data, it will generate rules which represents non-temporal association between attributes. In this way, data after processing $w$-moving average is used to discover association with $w$ or longer time-effect. Ideally, from $w=\infty$ down to $w=1$, we decompose all the independent time-effect associations between attributes. However, the timeconstant in which users are interested will be limited and the moving average method shown in Section 3 uses a set of $w$ given by users. Thus, application of rule discovery to each table will generate a sequence of temporal associations between attributes. If some temporal associations will be different from associations with $w=\infty$, then these specific relations will be related with a new discovery.

\subsection{Summary of Second Preprocessing and Rule Discovery}

Second preprocessing method and rule discovery are summarized as follows. 
1. Calculate $\hat{y}_{\infty}$ and $\sigma_{\infty}$ from the table of $w=\infty$;

2. Repeat for each $w$ in List $L_{w}$;

( $w$ is sorted in a descending order.)

a) Select a table of $w: T_{w}$;

i. Standardize continuous and ranking attributes;

ii. Calculate qualitative trends for continuous and ranking attributes;

iii. Construct a new table for qualitative trends;

iv. Apply rule discovery method for temporal sequences;

b) Apply rule induction methods to the original table $T_{w}$;

\section{Experimental Results}

The above rule discovery system is implemented in CEARI(Combination of Extended Moving Average and RUle Induction). CEARI was applied to a clinical database on motor neuron diseases, which consists of 1477 samples, 3 classes. Each patient is followed during 15 years. A list of $w,\{w\}$ was set to $\{10$ years, 5 years, 1 year, 3 months, 2 weeks $\}$ and thresholds, $\delta_{p(D \mid R)}$ and $\delta_{p(R \mid D)}$ were set to 0.60 and 0.30 ,respectively.

One of the most interesting problems of Motor neuron diseases (MND) is how long it takes each patient to suffer from respiratory failure, which is the main cause of death 3 It is empirically known that some types of MND is more progressive than other types and that their survival period is much shorter than others. The database for this analysis describes all the data of patients suffering from MND.

Non-temporal Knowledge. The most interesting discovered rules are:

$$
\begin{aligned}
& {[\text { Major_Pectolis }<3] \rightarrow\left[\mathrm{PaCO}_{2}>50\right](P(D \mid R): 0.87, P(R \mid D): 0.57),} \\
& {[\text { Minor_Pectolis }<3] \rightarrow\left[\mathrm{PaO}_{2}<61\right] \quad(P(D \mid R): 0.877, P(R \mid D): 0.65) .}
\end{aligned}
$$

Both rules mean that if some of the muscles of chest, called Major Pectolis and Minor Pectolis are weak, then respiratory function is low, which suggests that muscle power of chest is closely related with respiratory function, although these muscles are not directly used for respiration.

Short-Term Effect. Several interesting rules are discovered:

$$
\begin{gathered}
{[\text { Major_Pectolis }=2] \rightarrow\left[\mathrm{PaO}_{2}: D\right]} \\
\quad(P(D \mid R): 0.72, P(R \mid D): 0.53, w=3 \text { (months })), \\
{[\text { Biceps }<3] \rightarrow\left[P a O_{2}: A 2\right]} \\
\quad(P(D \mid R): 0.82, P(R \mid D): 0.62, w=3 \text { (months })) . \\
{[\text { Biceps }>4] \rightarrow\left[P a O_{2}: Z A\right]} \\
(P(D \mid R): 0.88, P(R \mid D): 0.72, w=3 \text { (months })) .
\end{gathered}
$$

\footnotetext{
3 The prognosis of MND is generally not good, and most of the patients will die within ten years because of respiratory failure. The only way for survival is to use automatic ventilator 2 .
} 
These rules suggest that if the power of muscles around chest is low, then respiratory function will decrease within one year and that if the power of muscles in arms is low, then respiratory function will decrease within a few years.

Long-Term Effect. The following interesting rules are discovered:

$$
\begin{aligned}
& {[\text { MajorPectolis }: A 3] \wedge[\text { Quadriceps }: A 3] \rightarrow\left[\mathrm{Pa}_{2}: A 3\right]} \\
& \quad(P(D \mid R): 0.85, P(R \mid D): 0.53, w=1(\text { year })), \\
& {[\text { Gastro }: A 3] \rightarrow\left[P a O_{2}: A 3\right]} \\
& (P(D \mid R): 0.87, P(R \mid D): 0.52, w=1(\text { year })) .
\end{aligned}
$$

These rules suggest that if the power of muscles of legs change very slowly, then respiratory function will decrease very slow. In summary, the system discovers that the power of muscles around chest and its chronological characteristics are very important to predict the respiratory function and how long it takes for a patient to reach respiratory failure.

\section{References}

1. Abiteboul, S., Hull, R., and Vianu, V. Foundations of Databases, Addison-Wesley, New York, 1995.

2. Adams, R.D. and Victor, M. Principles of Neurology, 5th edition, McGraw-Hill, NY, 1993.

3. Agrawal, R., Imielinski, T., and Swami, A., Mining association rules between sets of items in large databases, in Proceedings of the 1993 International Conference on Management of Data (SIGMOD 93), pp. 207-216, 1993.

4. Das, G., Lin, K.I., Mannila, H., Renganathan, G. and Smyth, P. Rule discovery from time series. In: Proceedings of Fourth International Conference on Knowledge Discovery and Data Mining, pp.16-22, 1998.

5. Fayyad, U.M., et al.(eds.)., Advances in Knowledge Discovery and Data Mining, AAAI Press, 1996.

6. Hamilton, J.D. Time Series Analysis, Princeton University Press, 1994.

7. Langley, P. Elements of Machine Learning, Morgan Kaufmann, CA, 1996.

8. Miksch, S., Horn, W., Popow, C., and Paky, F. Utilizing temporal data abstraction for data validation and therapy planning for artificially ventilated newborn infants. Artificial Intelligentce in Medicine, 8, 543-576, 1996.

9. Tsumoto, S. and Tanaka, H., PRIMEROSE: Probabilistic Rule Induction Method based on Rough Sets and Resampling Methods. Computational Intelligence, 11, 389-405, 1995.

10. Tsumoto, S. Knowledge Discovery in Medical MultiDatabases: A Rough Set Approach, Proceedings of PKDD99(in this issue), 1999.

11. Van Bemmel,J. and Musen, M. A. Handbook of Medical Informatics, SpringerVerlag, New York, 1997. 